\title{
Point-of-Care Ultrasound-Guided Conservative Fluid Therapy Improved the Outcome of Critically III Patients: A Before-and-After Study
}

Hui He Jinniu Hospital affiliated to Sichuan Provincial Hospital

Mingqiang Zeng

Jinniu Hospital affiliated to Sichuan Provincial Hospital

Jing Chen

Jinniu Hospital affiliated to Sichuan Provincial Hospital

Lei Deng

Jinniu Hospital affiliated to Sichuan Provincial Hospital

Youdai Chen ( $\nabla$ chenyoudai@sohu.com )

Jinniu Hospital affiliated to Sichuan Provincial Hospital

\section{Research Article}

Keywords: Ultrasound, Internal jugular vein, Inferior vena cava, Fluid therapy, Critically ill

Posted Date: May 17th, 2021

DOI: https://doi.org/10.21203/rs.3.rs-133531/v2

License: (c) (i) This work is licensed under a Creative Commons Attribution 4.0 International License.

Read Full License 


\section{Abstract}

\section{Objectives}

To study the impact of fluid balance on the outcome of critically ill patients.

\section{Methods}

Critically ill patients managed with point-of-care ultrasound were compared with those managed without. Distended internal jugular veins and inferior vena cava with reduced collapsibility were taken as signs of hypervolemia.

\section{Results}

Compared with critically ill patients admitted before application of point-of-care ultrasound assessment (from March, 2019 through October, 2019; 291 cases), cases admitted after (from November, 2019 through June, 2020; 285 cases) had significantly lower in-ICU mortality ( $34.7 \%$ vs $26.7 \%, p=0.038$; Fisher's exact test), together with a dramatic change from overall positive fluid balance to negative one (for cumulative fluid balance during ICU stay, $2820 \pm 1381 \mathrm{ml}$ vs $-10 \pm 39 \mathrm{ml} ; \mathrm{p}=0.001)$. Multiple logistic regression showed that cumulative fluid balance during ICU stay, Acute Physiology and Chronic Health Evaluation (APACHE) II score and Sequential Organ Failure Assessment (SOFA) score on admission were independent risk factors for in-ICU mortality ( $p<0.001, p<0.001$ and $p=0.043$ respectively). After controlling for disease severity, Cox hazard ratio of cases with a negative cumulative fluid balance during ICU stay was 0.683 (95\% confidence interval 0.475-0.981; $p=0039)$.

\section{Conclusions}

Negative cumulative fluid balance during ICU stay was associated with a reduced in-ICU mortality.

\section{Introduction}

Fluid therapy was of paramount importance in the management of critically ill patients. Fluids in excessive amounts could have deleterious effects on several organ functions, including acute kidney injury and worsening respiratory function[1]. The danger of volume overload had been noted in some critically ill patients, namely sepsis/septic shock patients[2-4]. However, the focus of intensivists was usually limited to resuscitation phase immediately after hospitalization of critically ill patients; intensivists paid little attention to fluid balance after initial hemodynamic stabilization. Fluid removal or de-resuscitation after hemodynamic stabilization was seldom put into practice[4,5]. And too often, fluid balance of critically ill patients other than sepsis/septic shock cases was not the concern of intensivists. Up to date, the danger of volume overload was not well understood and appreciated.

In previous studies, internal jugular vein was found to be a good surrogate of central venous pressure (CVP)[6-11]. Distention of internal jugular veins had the same implications as distention of superficial 
neck veins, and the only difference was that ultrasound could probe deeper. Although visual inspection of jugular veins as an estimate of CVP had been an integral part of physical examination, its major limitation was that jugular veins were not always observable, especially in obese patients[12]. Although the disagreement persisted as to the interpretation of size and collapsibility of inferior vena cava[13-16], distension and reduced collapsibility of inferior vena cava pointed to normo-/ hyper-volemia and were proved to be very beneficial in guiding fluid therapy[17]. The present study was carried out to compare the outcome of critically ill patients before and after the implementation of point-of-care ultrasound examination of both internal jugular veins and inferior vena cava.

\section{Results}

From March, 2019 through June, 2020, 576 cases were enrolled. Cases enrolled before application of point-of-care ultrasound were not significantly different from those enrolled after application of ultrasound, in respect to age, principal diagnoses, and APACHE II and SOFA scores on admission (Table 1).

On admission, more than half cases had a serum BNP $>400 \mathrm{pg} / \mathrm{ml}$ or in the gray zone (between 100 and $400 \mathrm{pg} / \mathrm{ml}$ ). $40 \%$ cases had a peak serum BNP $>400 \mathrm{pg} / \mathrm{ml}$. Both serum BNP on admission and peak serum BNP of non-survivors were significantly higher than that of survivors (Fig 1).

Cumulative fluid balance during ICU stay for cases enrolled before application of ultrasound was significantly more than that for cases enrolled after application of ultrasound; there was a dramatic change from overall positive fluid balance to negative one (Fig 2). After application of point-of-care ultrasound, intravenously-administered furosemide was markedly increased; significantly more furosemide was administered intravenously during the first three days to attain a negative fluid balance during ICU stay (Fig 3). In-ICU mortality of cases enrolled before application of ultrasound was significantly higher than that of cases enrolled after application of ultrasound ( $34.7 \%$ vs $26.7 \%, p=0.038$; Fisher's exact test); cases with positive cumulative fluid balance during ICU stay had a markedly higher inICU mortality than those with negative one (Fig 4).

After controlling for APACHE II and SOFA scores on admission, Cox hazard ratio of cases with a negative cumulative fluid balance during ICU stay was 0.683 (95\% confidence interval $0.475-0.981 ; p=0039$ ). Multiple logistic regression showed that cumulative fluid balance during ICU stay, APACHE II score and SOFA score on admission, not day 2 and day 3 cumulative fluid balance, were independent risk factors for in-ICU mortality. APACHE II score and SOFA score on admission was not collinear; condition index was $<10$ (8.67; linear regression). Studentized residuals was <2 (Fig 5). APACHE II and SOFA scores on admission, cumulative fluid balance during ICU stay, day 2 and day 3 cumulative fluid balances all could predict in-ICU mortality (Fig 6).

Compared with cases before application of point-of-care ultrasound, cases after application of ultrasound had significantly shorter length of ventilator support, $4.7 \pm 0.7$ days vs. $2.8 \pm 0.2$ days $(Z=2.465$, 
$p=0.014$; Mann-Whitney $U$ test). Their LOSs were not significantly different, $8.4 \pm 0.8$ days vs. $6.5 \pm 0.3$ days ( $Z=0.195, p=0.845$; Mann-Whitney U test).

\section{Discussion}

In the present study, two senior physicians of the same ICU, with similar background, were sent to preside over ICU daily works of Jinniu Hospital affiliated to Sichuan Provincial Hospital. And the patients enrolled before application of point-of-care ultrasound were not significantly different from those enrolled after, in respect to principal diagnoses, disease severity on admission and age. These patients were comparable, except for the application of point-of-care ultrasound and physicians' fluid preference.

The serum BNP values of these patients suggested that more than half cases probably experienced congestive heart failure during ICU stay. Volume removal was of paramount importance in these cases.

The present study showed that cumulative fluid balance during ICU stay was an independent risk factor of in-ICU mortality. After application of point-of care ultrasound, a dramatic change from positive fluid balance to negative one occurred. And the change in fluid balance was associated with a significant reduction in in-ICU mortality. After controlling for APACHE II score and SOFA score on admission, negative cumulative fluid balance during ICU was protective.

It was reasonable to direct fluid therapy with point-of-care ultrasound examination of internal jugular veins and inferior vena cava. Point-of-care ultrasound examination of internal jugular veins should not be viewed as a brand-new concept; distention of internal jugular veins had the same implications as distention of superficial neck veins. Point-of-care ultrasound examination of internal jugular veins was better than visual inspection of superficial neck veins, in that it could probe deeper. One major limitation of visual inspection was that jugular veins were not always observable, especially in obese patients[12]. Distension and reduced collapsibility of inferior vena cava pointed to normo-/ hyper-volemia and were proved to be very beneficial in guiding fluid therapy[17].

Why cumulative fluid balance during ICU stay notably affected the outcome of critically ill patients? There were two reasons worth mentioning. First, some critically ill patients were hypervolemic from the very beginning of their hospitalization. Second, catabolism and disuse.

Previous studies showed that many critically ill patients were essentially hypervolemic. For example, in critically ill anemic patients, hypervolemia was common[18]. "Plasma volume excess", in addition to "hemoglobin deficiency", often characterized anemia when plasma volume was measured[19]. Plasma volume could increase by $70 \%$ in chronic severe anemia[20]. In heart failure with preserved ejection fraction, expanded total blood volume together with true anemia was common[21, 22]. In volume overload decompensated chronic heart failure, plasma volume expansion dilution-related "anemia" predominated[23]. Dilution in consequence of sodium and water retention was an important reason for development of anemia in chronic heart failure[24]. In patients with chronic kidney disease, anemia was often complicated with fluid retention and overhydration, resulting in increased risk of cardiovascular 
morbidity and mortality and chronic kidney disease progression[25]. Expansion of plasma volume and hemodilution were important features of anemia due to hemopoietic diseases, such as leukemia[26]. In the present study, many patients had elevated serum BNP on admission.

Most critically ill patients experienced a catabolic course which was not easily reversed by medication or nutritional support. Critically ill patients developed muscle atrophy rapidly[27], as a result of both anabolic resistance (reduced stimulation of muscle protein synthesis to a given dose of protein/amino acids) and enhanced skeletal muscle breakdown[28], or as a result of iatrogenic protein undernutrition[29], and preferentially affecting the lower limbs[30]. Both catabolism and disuse in bedridden cases resulted in breakdown of proteins and reduction of dry body weight, leading to production of "endogenous water" and release of water from degraded proteins and fats. Fluid derived from catabolism and disuse should be removed to avoid volume overload and tissue edema.

In critically ill postoperative patients and patients with severe nutritional depletion, a dramatic change in body composition was observed. Reduction of body weight was accompanied by a marked increase in extracellular water (by 4.0-5.1 liters in 31-34 days) [31]. Rapid and massive total protein loss was accompanied by retention of large amounts of fluids in critically ill patients with major traumatic injury or severe sepsis. Trauma patients had retained $>6 \mathrm{~L}$ and sepsis patients $>12 \mathrm{~L}$ of fluids, mainly in the extracellular compartment, by the time they were hemodynamically stable. And in elderly patients $>60$ years) the period of extracellular water expansion was markedly prolonged compared to younger patients $(<40$ years)[32].

Fluids in excessive amounts could have deleterious effects on several organ functions, including acute kidney injury and worsening respiratory function[1]. The danger of volume overload was already noted in some critically ill patients, namely sepsis/septic shock patients[2-4]. The present study was in line with these studies, suggesting that conservative fluid strategy was superior to liberal one, and that fluid removal or de-resuscitation after hemodynamic stabilization should be put into practice.

The negative impact of positive cumulative fluid balance could not be overestimated. Many hypervolemic patients died when given diuretics. If fluid management could be improved, these cases would add to the difference in survival between cases enrolled before application of point-of-care ultrasound and those enrolled after. The diuresis of these deceased patients was not well titrated, in respect of timing and dosing of diuretic administration. Admittedly, the fluid balance was difficult to maintain in these critically ill patients. Studies conducted by nephrologists showed that shift of fluid between intravascular and extravascular compartments could be surprisingly rapid; plasma refill rate reached up to $20.1 \pm 4.0$ $\mathrm{ml} /$ min during hemodialysis[33-35]. When re-absorption far exceeded the rate of volume removal, a "well-controlled" pace of negative fluid balance would result in hemodynamic instability.

One main problem was that physicians had difficulty adapting to such a paradigm shift. For some physicians, fluid challenge and positive fluid balance were still the only solution when facing shock and lactic acidosis. The improvement in outcome would be greater if all physicians had abandoned fluid preference. 
Another limitation of the present study was that 28-day or 90-day mortality was not recorded. However, the contribution of post-ICU treatment to 28-day or 90-day mortality was difficult to estimate, and physicians' and surgeons' fluid preferences would have major impact on these outcome measures.

In conclusion, volume overload might become increasingly evident in the course of illness. If not curbed, volume overload would minimize the chance of survival of critically ill patients. Overgenerous fluid administration should be avoided, and fluid balance should be meticulously managed during the whole ICU stay. More studies are needed to address the importance of fluid management during the whole ICU stay.

\section{Methods}

We conducted this study in Intensive Care Unit (ICU) of Jinniu Hospital affiliated to Sichuan Provincial Hospital. This ICU was a 24-bed setting for medical and surgical patients. This hospital had no anesthesia recovery room and emergency intensive care unit. The Ethical Committee of Jinniu Hospital affiliated to Sichuan Provincial Hospital approved the study protocol. The informed consents were obtained from the patients or their close relatives. The study was conducted in accordance with the Declaration of Helsinki.

From March, 2019 on, senior physicians from Emergency Intensive Care Unit (EICU) of Sichuan Provincial Hospital were sent to preside over daily works of this ICU, first by Dr Deng for 8 months, then by Dr Chen (the corresponding author) for another 8 months. During the first 8 months, point-of-care ultrasound was seldom used. Point-of-care ultrasound was widely used during the late 8 months. Distended internal jugular veins and inferior vena cava were viewed as signs of hypervolemia, together with raised CVP or brain natriuretic peptide (BNP) or radiological/clinical findings of pulmonary edema.

Cross-sectional area of internal jugular vein and diameter of inferior vena cava were measured with Mindray M9 (Shenzhen Mindray Bio-medical Electronics Co., Ltd., Shenzhen, Guangdong Province, China). Internal jugular vein was measured at cricoid level. Inferior vena cava was measured just below the xyphoid process, and within $2 \mathrm{~cm}$ from right atrium. They were both measured at end-expiration. To measure internal jugular vein, the probe was gently placed on the skin; internal jugular vein was readily deformed by any pressure applied to it. Cross-sectional areas of internal jugular veins were measured because many of them were in irregular shapes, and only a small number of them were rounded. Patients were routinely assessed with point-of-care ultrasound within $10 \mathrm{hrs}$ after admission. Collapsibility index of inferior vena cava was assessed with M-mode ultrasound. Whenever distended internal jugular vein with a collapsibility index (cross-sectional area) $<50 \%$ and/or inferior vena cava diameter $>15 \mathrm{~mm}$ with a collapsibility index(diameter) $<50 \%$ were detected, negative fluid balance became a goal. Different from previous studies[13-17], distended inferior vena cava with reduced collapsibility index was taken as a sign of hypervolemia, not a sign of lack of fluid responsiveness. Loop diuretics were frequently used to achieve a negative fluid balance, together with fluid restriction, and renal replacement therapy when indicated. Patients were re-assessed daily until becoming hemodynamically stable. 
Inclusion criteria: a successive cohort of patients admitted to ICU were included.

Exclusion criteria: patients died or discharged against medical advice within $10 \mathrm{hrs}$ after admission or referral to ICU; hemodynamically stable surgical patients, either emergent or elective, referred to EICU for anesthesia recovery only, experiencing an uneventful recovery, and with an ICU stay shorter than 3 days. These surgical patients were referred to ICU simply because there was no anesthesia recovery room. All patients with an ICU stay <' 10 hrs had a positive fluid balance.

Outcome measures: The primary endpoint was in-ICU mortality. The survivors were referred to medical or surgical ward. Non-survivors included patients brought home on hospice, to be with the family before death. It was stipulated by local authority that patients died in hospitals had to be cremated. Some patients were brought home before death because they wanted to be buried, not cremated. They died on the way home or immediately back home. Non-survivors also included patients referred to Sichuan Provincial Hospital and died within $72 \mathrm{hrs}$ after referral. The secondary endpoints were length of ICU stay (LOS), length of ventilator support (invasive and/or non-invasive).

APACHE (Acute Physiology and Chronic Health Evaluation) II and SOFA (Sequential Organ Failure Assessment) scores were calculated by the corresponding author (YC) alone, using data of the first $24 \mathrm{hrs}$ after admission to EICU. For patients died within $24 \mathrm{hrs}$ after admission, data at least $30 \mathrm{~min}$ prior to death were taken into consideration.

Gastrointestinal loss and third space loss which was drawn with a needle and syringe or percutaneously drained were counted as output. Insensible losses were not counted.

Fluid balance=intravenous fluid+oral intake-urine output-gastrointestinal loss-third space drainage.

\section{Statistical analysis}

These data were analyzed with IBM SPSS Statistics 20. APACHE II and SOFA scores of different groups were compared with Student's $t$-test. In-ICU mortalities of different groups were compared with chi-square test and Fisher's exact test. Fluid balances of cases enrolled before application of point-of-care ultrasound were compared with that of cases enrolled after application of ultrasound using Student's $t$ test. Amounts of intravenously-administered furosemide before application of point-of-care ultrasound were compared with that after application of ultrasound using Mann-Whitney $U$ test. Serum brain natriuretic peptide (BNP) of survivors was compared with that of non-survivors using Mann-Whitney $U$ test. Logistic regression analysis was conducted to find out the risk factors of in-ICU mortality among APACHE II score, SOFA score, day 1 fluid balance, day 2 cumulative fluid balance, day 3 cumulative fluid balance and cumulative fluid balance during ICU stay. Logistic regression analysis did not report odds ratio and confidence interval when fluid balances were in $\mathrm{ml}$. Then logistic regression analysis was done after fluid balances were transformed into - Ls (liters). Before logistic regression analysis, collinearity diagnosis was done for APACHE II score and SOFA score on admission with linear regression analysis. Survival curve (Cox regression model) and ROC (receiver operating characteristic) curve were plotted with 
IBM SPSS Statistics 20. Length of ventilator support and LOS of cases before application of ultrasound were compared with that of cases after application of ultrasound using Mann-Whitney $U$ test.

\section{Abbreviations}

ICU, intensive care unit; APACHE, Acute Physiology and Chronic Health Evaluation; SOFA, Sequential Organ Failure Assessment; CVP, central venous pressure; LOS, length of ICU stay; BNP, brain natriuretic peptide; ROC, receiver operating characteristic; AUC, area under curve.

\section{Declarations}

\section{Ethnics approval and consent to participate}

The ethical committee at Jinniu Hospital approved the study protocol. The informed consents were obtained from the patients or their close relatives.

\section{Consent for publication}

Not applicable.

\section{Competing interests}

The authors declare that they have no competing interests.

\section{Availability of data and material}

The datasets during and/or analyzed during the current study are available from the corresponding author on request.

\section{Authors' contributions}

$\mathrm{HH}$ and DL took part in data collection and analysis. ZM conducted ultrasound examination. CJ took part in data collection. $\mathrm{CY}$ took part in data collection and analysis, and wrote the manuscript. All authors read and approved the final manuscript.

\section{Funding}

Dr Y Chen was supported by a grant from Sichuan Provincial Health Administration (416001004078008).

\section{References}

1. Besen, B. A., Gobatto, A. L., Melro, L. M., Maciel, A. T. \& Park, M. Fluid and electrolyte overload in critically ill patients: An overview. World. J Crit Care Med. 4 (2), 116-129 (2015). 
2. Marik, P. \& Bellomo, R. A rational approach to fluid therapy in sepsis. Br. J Anaesth. 116 (3), 339-349 (2016).

3. Mitchell, K. H. et al. Volume overload: prevalence, risk factors, and functional outcome in survivors of septic shock. Ann. Am Thorac Soc. 12, 1837-1844 (2015).

4. Malbrain, M. L. et al. Fluid overload, de-resuscitation, and outcomes in critically ill or injured patients: a systematic review with suggestions for clinical practice. Anaesthesiol. Intensive Ther. 46, 361-380 (2014).

5. Bissell, B. D. et al. Impact of protocolized diuresis for de-resuscitation in the intensive care unit. Crit. Care. 24, 70 (2020).

6. Hossein-Nejad, H., Mohammadinejad, P. \& Ahmadi, F. Internal jugular vein/common carotid artery cross-sectional area ratio and central venous pressure. J. Clin Ultrasound. 44 (5), 312-318 (2016).

7. Hilbert, T., Ellerkmann, R. K., Klaschik, S., Putensen, C. \& Thudium, M. The use of internal jugular vein ultrasonography to anticipate low or high central venous pressure during mechanical ventilation. $J$. Emerg Med. 50 (4), 581-587 (2016).

8. Bailey, J. K., McCall, J., Smith, S. \& Kagan, R. J. Correlation of internal jugular vein/common carotid artery ratio to central venous pressure: a pilot study in pediatric burn patients. J. Burn Care Res. 33 (1), 89-92 (2012).

9. Siva, B., Hunt, A. \& Boudville, N. The sensitivity and specificity of ultrasound estimation of central venous pressure using the internal jugular vein. J. Crit Care. 27 (3), 3157-3111 (2012).

10. Donahue, S. P., Wood, J. P., Patel, B. M. \& Quinn, J. V. Correlation of sonographic measurements of the internal jugular vein with central venous pressure. Am. J Emerg Med. 27 (7), 851-855 (2009).

11. Nik Muhamad, N. A., Safferi, R. S. \& Robertson, C. E. Internal jugular vein height and inferior vena cava diameter measurement using ultrasound to determine central venous pressure: a correlation study. Med. J Malaysia. 70 (2), 63-66 (2015).

12. Lipton, B. Estimation of central venous pressure by ultrasound of the internal jugular vein. $A m$. J Emerg Med. 18 (4), 432-434 (2000).

13. Laborda, A. et al. Influence of breathing movements and Valsalva maneuver on vena caval dynamics. World J Radiol. 6 (10), 833-839 (2014).

14. Bodson, L. \& Vieillard-Baron, A. Respiratory variation in inferior vena cava diameter: surrogate of central venous pressure or parameter of fluid responsiveness? Let the physiology reply. Crit. Care. 16 (6), 181 (2012).

15. Kory, P. \& Counterpoint Should acute fluid resuscitation be guided primarily by inferior vena cava ultrasound for patients in shock? No. Chest. 151 (3), 533-536 (2017).

16. Schmidt, G. A. \& Point Should acute fluid resuscitation be guided primarily by inferior vena cava ultrasound for patients in shock? Yes. Chest. 151 (3), 531-532 (2017).

17. Prekker, M. E., Scott, N. L., Hart, D., Sprenkle, M. D. \& Leatherman, J. W. Point-of-care ultrasound to estimate central venous pressure: a comparison of three techniques. Crit. Care Med. 41 (3), 833-841 
(2013).

18. Van, P. Y. et al. Blood volume analysis can distinguish true anemia from hemodilution in critically ill patients. J. Trauma. 70 (3), 646-651 (2011).

19. Otto, J. M. et al. Hemoglobin concentration, total hemoglobin mass and plasma volume in patients: implications for anemia. Haematologica. 102 (9), 1477-1485 (2017).

20. Anand, I. S., Chandrashekhar, Y., Ferrari, R., Poole-Wilson, P. A. \& Harris, P. C. Pathogenesis of oedema in chronic severe anaemia: studies of body water and sodium, renal function, haemodynamic variables, and plasma hormones. Br. Heart J. 70, 357-362 (1993).

21. Miller, W. L. \& Mullan, B. P. Volume overload profiles in patients with preserved and reduced ejection fraction chronic heart failure: are there differences? A pilot study. JACC. Heart Fail. 4 (6), 453-459 (2016).

22. Noumi, B., Teruya, S., Salomon, S., Helmke, S. \& Maurer, M. S. Blood volume measurements in patients with heart failure and a preserved ejection fraction: implications for diagnosing anemia. Congest. Heart Fail. 17 (1), 14-18 (2011).

23. Miller, W. L. \& Mullan, B. P. Peripheral venous hemoglobin and red blood cell mass mismatch in volume overload systolic heart failure: implications for patient management. J. Cardiovasc Trans/ Res. 8 (7), 404-410 (2015).

24. Steinborn, W., Doehner, W. \& Anker, S. D. Anemia in chronic heart failure-frequency and prognostic impact. Clin. Nephrol. 60 (Suppl 1), S103-S107 (2002).

25. Hung, S. C. et al. Association of fluid retention with anemia and clinical outcomes among patients with chronic kidney disease. J. Am Heart Assoc. 4 (1), e001480 (2015).

26. Linderkamp, O., Lau, B., Riegel, K. P. \& Betke, K. Blood volume of children with leukemia. Acta. Paediatr Scand. 67 (3), 281-284 (1978).

27. Kawahara, K. et al. Evaluation of the site specificity of acute disuse muscle atrophy developed during a relatively short period in critically ill patients according to the activities of daily living level: a prospective observational study. Aust. Crit Care. 30 (1), 29-36 (2017).

28. Morton, R. W., Traylor, D. A., Weijs, P. J. M. \& Phillips, S. M. Defining anabolic resistance: implications for delivery of clinical care nutrition. Curr. Opin Crit Care. 24 (2), 124-130 (2018).

29. Phillips, S. M., Dickerson, R. N., Moore, F. A., Paddon-Jones, D. \& Weijs, P. J. Protein turnover and metabolism in the elderly intensive care unit patient. Nutr. Clin Pract. 32 (1-suppl), 112-120 (2017).

30. Turton, P., Hay, R., Taylor, J., McPhee, J. \& Welters, I. Human limb skeletal muscle wasting and architectural remodeling during five to ten days intubation and ventilation in critical care-an observational study using ultrasound. BMC. Anesthesiol. 16 (1), 119 (2016).

31. Elwyn, D. H., Bryan-Brown, C. W. \& Shoemaker, W. C. Nutritional aspects of body water dislocations in postoperative and depleted patients. Ann. Surg. 182 (1), 76 (1975).

32. Plank, L. D. Body composition studies in critical illness in Handbook of Anthropometry: Physical Measures of Human Form in Health and Disease (ed. Preedy, V.R.)2285-2298(Springer Science + 
Business Media, LLC, 2012).

33. Brummelhuis, W. J., van Gees, R. J., van Schelven, L. J. \& Boer, W. H. Sodium profiling, but not cool dialysate, increases the absolute plasma refill rate during hemodialysis. ASAIO. J. 55(6), 575580(2009).

34. Shulman, T., Heidenheim, A. P., Kianfar, C., Shulman, S. M. \& Lindsay, R. M. Preserving central blood volume: changes in body fluid compartments during hemodialysis. ASAIO. J. 47 (6), 615-618 (2001).

35. Marenzi, G. C. et al. Circulatory response to fluid overload removal by extracorporeal ultrafiltration in refractory congestive heart failure. J. Am Coll Cardiol. 38 (4), 963-968 (2001).

\section{Tables}

Table 1 Demographic characteristics of subjects

\begin{tabular}{|llll|}
\hline & $\begin{array}{l}\text { Before application } \\
\text { of ultrasound }\end{array}$ & $\begin{array}{l}\text { After application of } \\
\text { ultrasound }\end{array}$ & Sig. \\
\hline No. of cases & 291 & 285 & \\
\hline Age (years old) & $65.5 \pm 17.4(14 \llbracket 97)$ & $65.9 \pm 17(15 \rrbracket 96)$ & $\mathrm{p}=0.395$ \\
\hline Sex (male:female) & $189: 102$ & $169: 116$ & \\
\hline Principal diagnosis & & & \\
\hline Sepsis/septic shock & 129 & 119 & $\mathrm{p}=0.556$ \\
\hline $\begin{array}{l}\text { Cardiac arrest/arrythmia/acute coronary } \\
\text { syndrome/heart failure }\end{array}$ & 38 & 36 & $\mathrm{p}=0.901$ \\
\hline $\begin{array}{l}\text { Intracranial hemorrhage/cerebral } \\
\text { infarction }\end{array}$ & 36 & 43 & $\mathrm{p}=0.397$ \\
\hline $\begin{array}{l}\text { Traumatic brain injury } \\
\text { Multiple trauma }\end{array}$ & 35 & 24 & $\mathrm{p}=0.17$ \\
\hline APACHE II score on admission & 17 & 18 & $\mathrm{p}=0.863$ \\
\hline SOFA score on admission & $21.2 \pm 9.4$ & $21.6 \pm 9.2$ & $\mathrm{p}=0.368$ \\
\hline
\end{tabular}

Age, APACHE II score and SOFA score were compared with Student's $t$-test; principal diagnoses were compared with Pearson chi-square test.

\section{Figures}



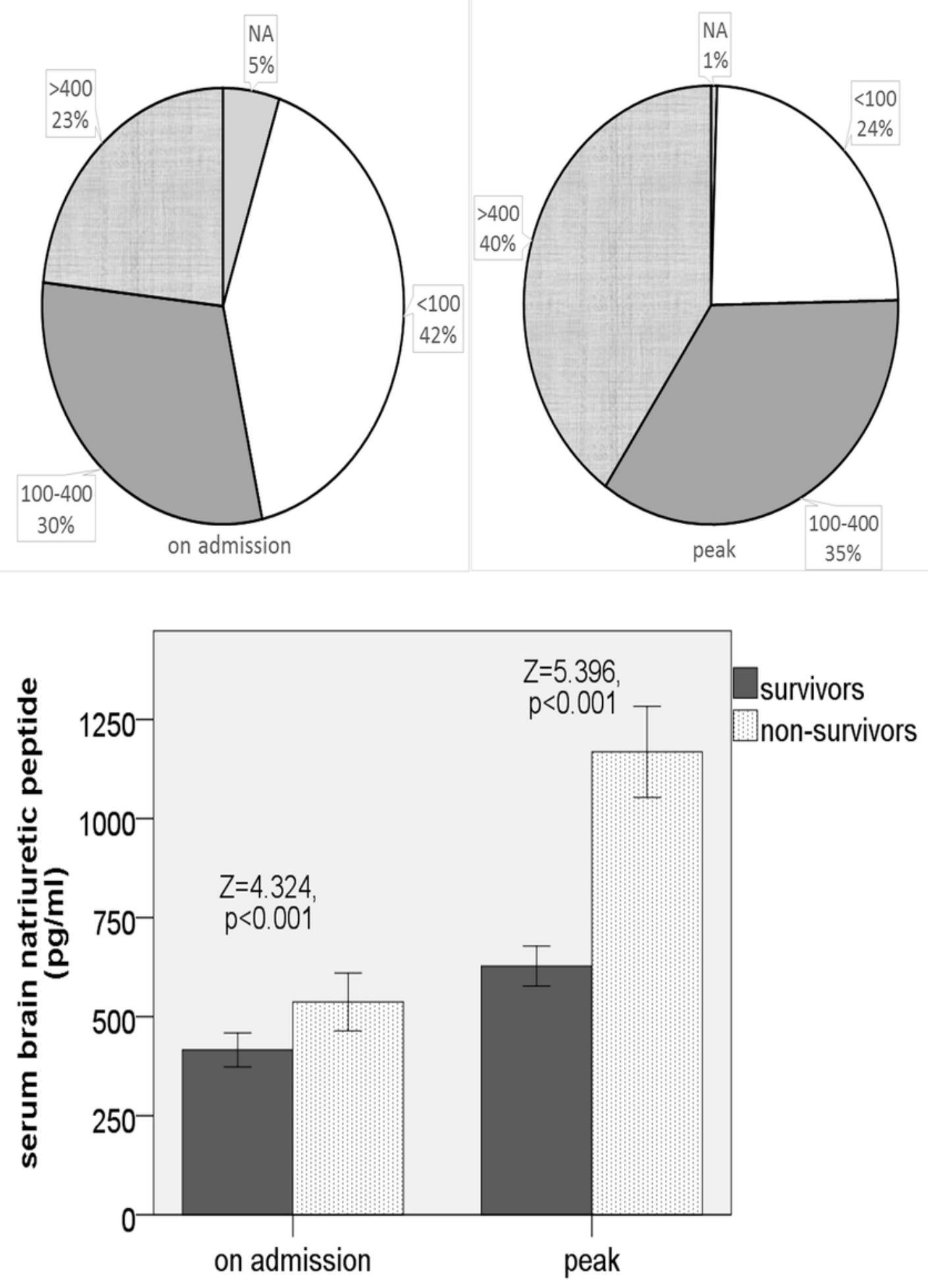

Figure 1

Serum BNP on admission and peak serum BNP. *Upper left: on admission; upper right: peak value. NA, not assayed. Serum BNP in pg/ml. Compared with Mann-Whitney U test. 


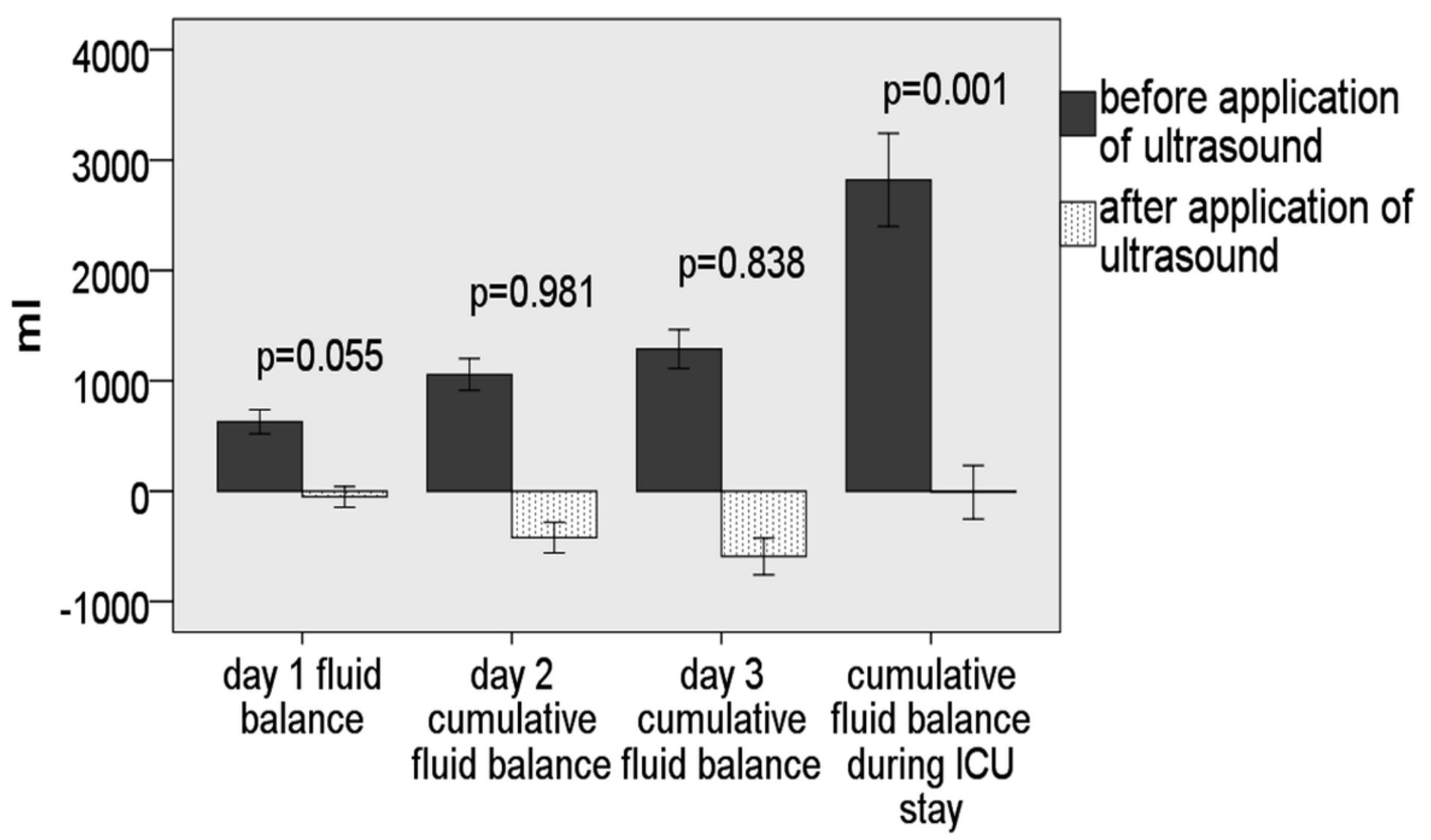

Figure 2

Fluid balances of the first three days and cumulative fluid balance during ICU stay. *Using Student's t-test. 

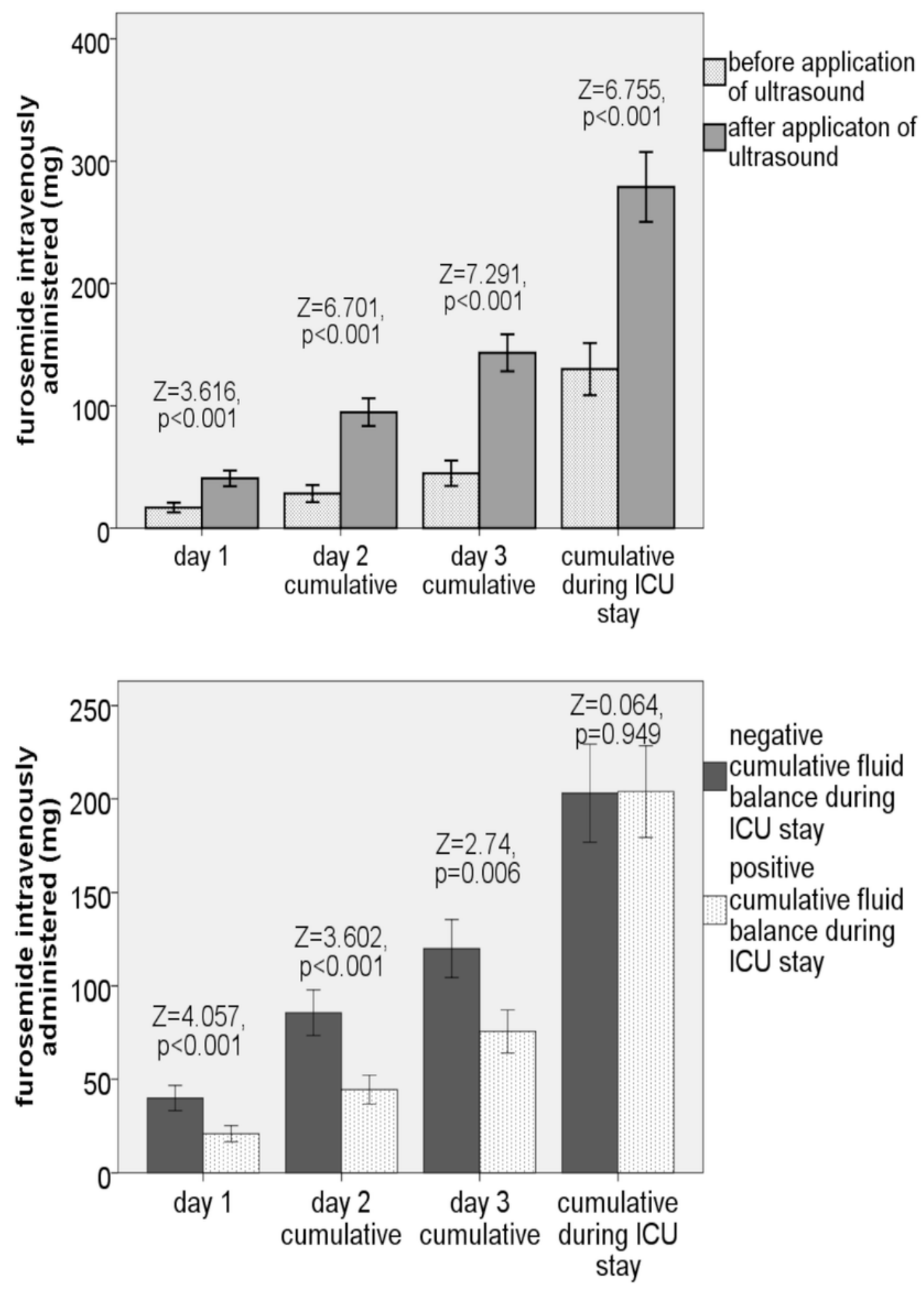

Figure 3

Amounts of furosemide intravenously administered. *Compared with Mann-Whitney U test. 


\section{in-ICU mortalities}

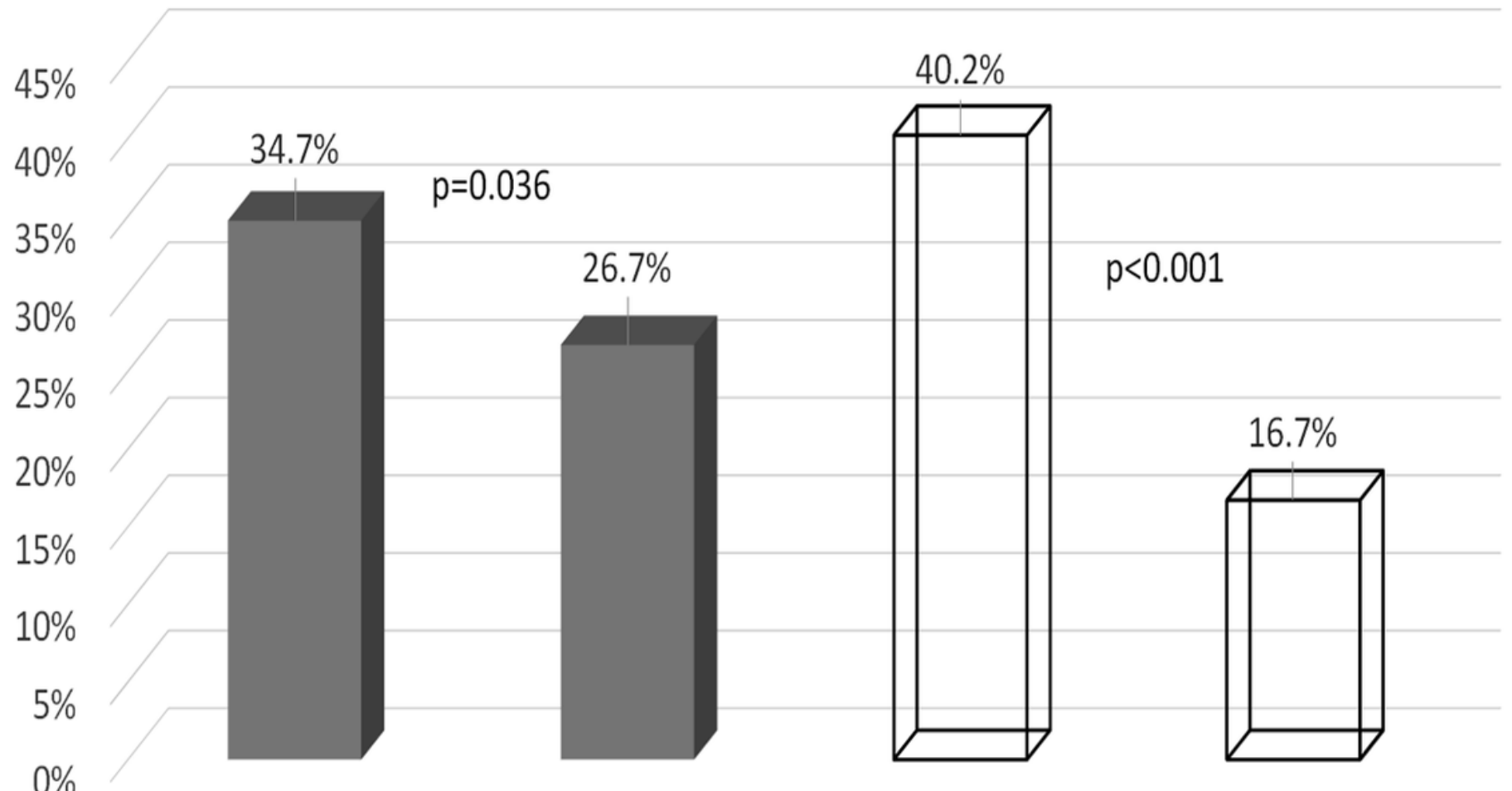

before application after application of positive cumulative negative cumulative of ultrasound ultrasound fluid balance during fluid balance during ICU stay ICU stay

\section{Figure 4}

In-ICU mortalities. ${ }^{*}$ Compared with chi-square test $(p=0.036)$ and Fisher's exact test $(p=0.038)$. For cases with positive vs. negative cumulative fluid balance during ICU stay, $p<0.001$ by both chi-square test and Fisher's exact test.
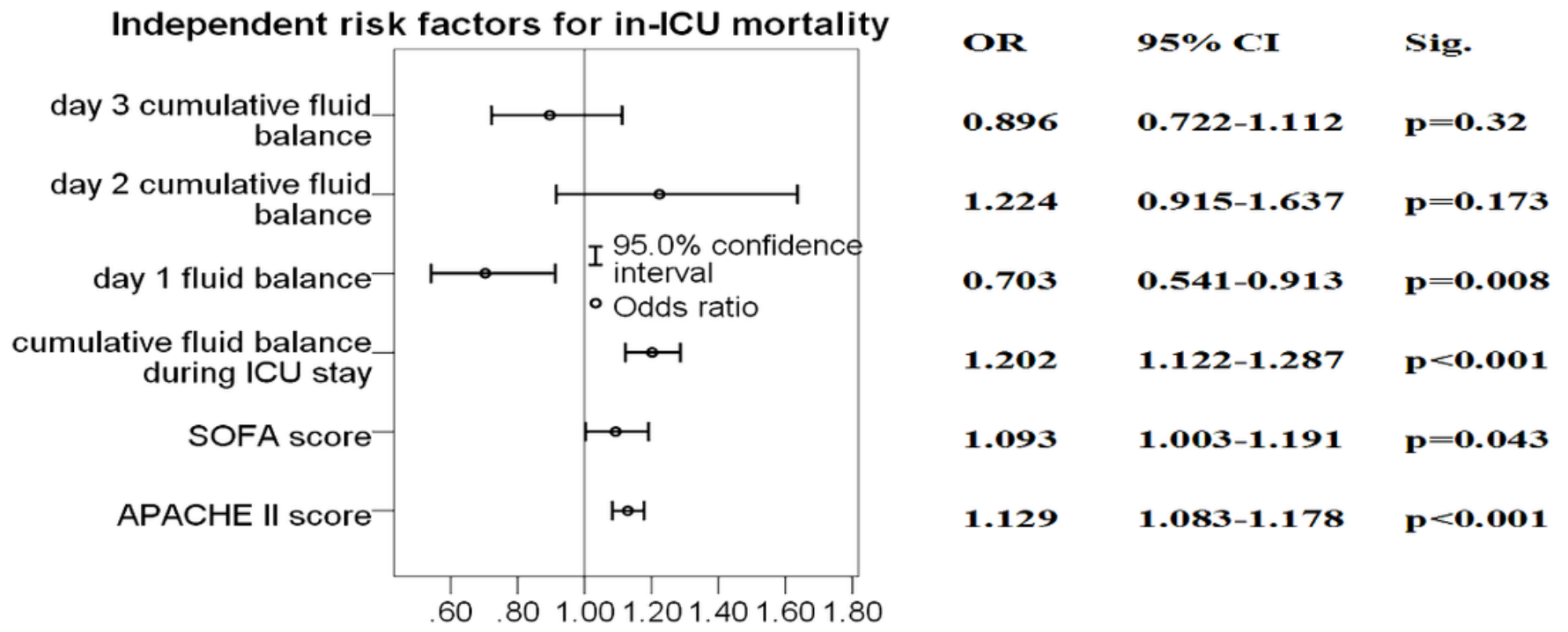
Figure 5

Multiple logistic regression analysis showing independent risk factors of in-ICU mortality. ${ }^{*} \mathrm{OR}$ for per liter of fluid. Studentized residuals $\leqq 0.971$.

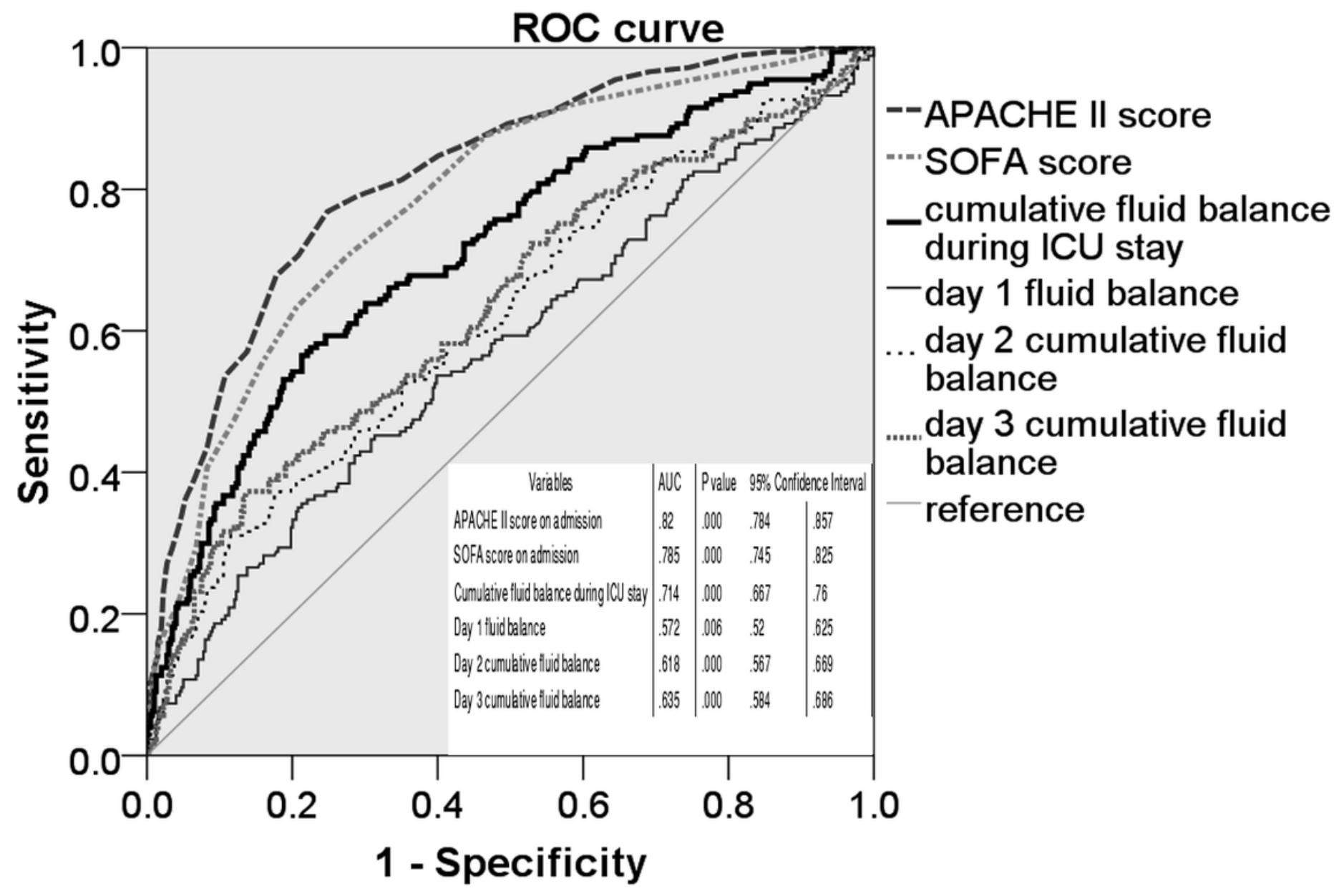

Diagonal segments are produced by ties.

Figure 6

Receiver operating characteristic (ROC) curves showing the relationship between sensitivity and 1specificity in determining APACHE II and SOFA scores, cumulative fluid balance during ICU stay, and fluid balances of the first three days to predict in-ICU mortality. 